industrial achievement. In deciding the best way to divide the very limited amount of land available so that it was used to the best advantage for the whole of Britain, it was essential to know exactly what one had to divide.

This brought the conference back to the point made by Sir George Pepler at the beginning of the conference, and which came up repeatedly in discussion, namely, the need for more accurate information about how land is actually being used in Great Britain-for, as Dr. Wibberly, of Wye College, pointed out at one point, even the figures of urban acreage are in dispute.

The conference therefore concluded by agreeing to forward the following resolution to the Council of the British Association: "That the British Association for the Advancement of Science represent to Her Majesty's Government the importance of arranging for the collection and publication of comprehensive statistics of the present use of all land in this country and to prepare an analysis on a national basis of the development plans now being submitted for approval in such a way that a balance sheet of land use may be drawn up. The Association submits that the collection of this factual information is a vital need in the present stage which town and country planning has reached in this country and in the formulation of national policies of land use". JoHN DAVY

\section{SOCIETY FOR THE STUDY OF FERTILITY}

\section{ANNUAL CONFERENCE}

$\mathrm{T}$ HE Society for the Study of Fertility held its annual conference during June 23-24 in the Anatomy Department of the University of Birming. ham. Prof. A. M. Daleq discussed some of the processes of synthesis occurring during the early stages of embryological development in rodents, as revealed by cytochemical studies. In the first of two stages which can be differentiated, there is a step-wise production of deoxyribonucleic acid. In the second stage, heralded by the differentiation of the embryonic knot, synthesis leads to the formation of granules rich in ribonucleic acid, which afterwards gives rise to specific proteins and the formation of enzymes. The enzymes at first are polyvalent; but later different specific enzymes appear. Embryonic development will be described more and more in terms of biochemical differentiation; at present, of course, it is possible to demonstrate this only in terms of cytochemistry. R. A. Beatty described experiments with rabbits designed to test the Russian hypotheses that heterospermic insemination (where semen from two or more donors is mixed prior to insemination) leads to specially beneficial effects among the offspring in respect of litter size and fœtal weight. The results he has obtained give no support whatever to these views, and it is concluded that the litters derived from heterospermic insemination are in no way superior to those resulting from homospermic insemination.

W. R. M. Morton has studied the effects on the mating behaviour of rats caused by section of the olfactory tracts and removal of the olfactory bulbs. He concludes that complete removal of the olfactory pathways interferes with the total mating reaction but does not entirely prevent effective copulation.
P. P. Scott and M. A. Lloyd-Jacob have made a study of reproduction in the domestic cat as maintained in an animal colony. It proved possible to follow the cestrous cycle by means of vaginal smears, and cestrus was found to occur at 12-14 days intervals, lasting 4-6 days. Typical œestrous behaviour was usually shown only in the presence of a tom cat, and the authors described a characteristic lateral flexion of the tail which was never observed except when œstrogens were present in significant amounts. Several consecutive matings during the period of œ.strus seemed to be necessary in order to lead to conception, and the average duration of pregnancy was 65 days. A remarkable excess of males over females in the litters born was found, the ratio being 133 males per 100 females at birth.

Further studies were reported from the Anatomy Department of the University of Liverpool, under the direction of Prof. R. G. Harrison, on male reproductive physiology. E. W. MacMillan and R. G. Harrison demonstrated that the ductus epididymidis possesses an inherent peristaltic activity essential to sperm propulsion by following radiographically the passage of 50 per cent barium sulphate solution injected into the rete testis. Within four days of injection, the mass occupies a position at the junction of body and tail, and by seven days it has reached the tail loop. The vas deferens is entered on the thirteenth day, and the genital tract is completely emptied by the fourteenth day. The rate of progress is unaffected by ligation of the vasa efferentia or by ipsilateral orchidectomy. R. Harris and R. G. Harrison have investigated the effect of low temperature on the guinea pig testis. They found that little permanent damage resulted, provided the temperature within the testis did not fall below $0^{\circ} \mathrm{C}$., and that freezing was more damaging than supercooling. Below that temperature occlusive spasm of the testicular artery occurred. Severe spermatogenic damage resulted from exposure of the testis to $-3^{\circ} \mathrm{C}$. for one hour, but a temperature of $-10^{\circ} \mathrm{C}$. was necessary to ensure an intratesticular temperature of $-4^{\circ} \mathrm{C}$. when the scrotum was frozen. Severe spermatogenic destruction led to a 90 per cent fall in testicular volume.

T. D. Glover described the effects of scrotal insulation, as a means of raising the testicular temperature, on the semen of the ram. Five days elapsed before any changes in the semen appeared, and then an increased proportion of coiled tails was seen. Following this, separation of heads and tails was observed, and a marked fall in the sperm density took place about two weeks after the commencement of the scrotal insulation. Insulation for a period of only twenty-four hours led to a brief change in the semen about twenty days later, but, after a further four days, the normal semen picture was resumed.

M. W. H. Bishop discussed the inter-relationships of semen characteristics in the bull. Conception-rate was found to be most closely correlated with impedance change frequency and inversely with the percentage of dead sperms. The most significant conclusion, however, arising from his study was that a large amount of variation in fertility could not be related to any of the numerous semen characteristics examined. E. G. Bassett gave a review of investi. gations recently carried out at the Ruakura Animal Research Station in New Zealand on problems related to fertility in farm animals.

A number of papers on human fertility were given by workers at the Royal Free Hospital, London. 
Mary Barton has found a high frequency of female infertility in couples where the husband was sterile and donor artificial insemination was contemplated. The high rate of failure of such insemination in women who, by the usual standards of investigation were regarded as fertile (and for whom, of course, apparently highly fertile semen was used), was also noticeable. Sara Field-Richards and M. Moore White described the use of local cestrogen therapy for uterine hypoplasia, and Phyllis Wade reported a pregnancy in a woman who had suffered from amenorrhœa following radiotherapy for ankylosing spondylitis.

G. I. M. Swyer reported a statistical assessment of post-coital test results which confirms the diagnostic, and to a lesser extent prognostic, value of the test. The not infrequent discrepancy between post-coital test results and semen examination means that the former cannot be regarded as a redundant step in infertility investigation. Clare Harvey discussed cytological events in the human testis, as revealed by biopsies in subfertile men, in relation to abnormalities in sperm morphology. She found that a high proportion of pear-shaped heads was correlated with subfertility and that these pear-headed spermatozoa developed as a result of abnormsil maturation of spermatogonia. Claude Taylor reported a conception following the insemination of centrifuged urine passed after intercourse by a man who was suffering from retrograde vesicular ejaculation. Oral treatment with potassium citrate maintained an alkaline urine in which spermatozoal activity persisted. Vigorous spermatozoal activity was found, so long as the urine $p H$ was close to 7 , but when either too acid or too alkaline, the spermatozoa failed to survive.

Three films were shown: one was on social behaviour in rhesus monkeys, by M. R. A. Chance; another, by H. G. Hanley, demonstrated a simplified and apparently effective technique for the operation of epididymo-vasostiomy ; and the third, by Prof. Richard J. Blandau, was on reproduction in the rat.

\section{INTERMETALLIC SEMICONDUCTORS}

$\mathrm{A}^{\mathrm{s}}$ LTHOUGH some of the earliest semiconductors of practical importance were chemical compounds, research interest in later years has been mainly in the elements germanium and silicon, partly due to the importance of transistor technology and partly because the problems of obtaining high purity and crystalline perfection appeared to be easier in a monatomic solid, and the theoretical interpretation more promising. However, with the extension of applications, new combinations of properties are desired, and it has been necessary to consider new materials-in particular, compounds, as there is little scope among the remaining elements. This trend was stimulated by the work of Welker in 1952 on compounds of the zinc-blende structure (isomorphous with germanium, but with alternate sites occupied by the respective elements). These were shown to have the high electron mobility characteristic of germanium and silicon which is essential for many applications. Still other types of compound may also have this property, so that the search has not been confined to zinc-blende types: generally, the group of compounds known as 'intermetallic' (that is, between elements having metallic properties, although the limits are not clearly defined) have shown the most interesting properties, and these are now being studied in a number of laboratories.

A study of compound semiconductors brings new problems of purification and crystal growing. The properties have then to be determined and interpreted in terms of theory, which may have to be extended or adapted in the process. Generalizations have to be attempted in order to guide further work. Finally, new applications of particular materials have to be considered. These points formed the theme of a symposium on intermetallic semiconductors which was held during April 26-27 at the Services Electronics Research Laboratory, Baldock, Herts, attended by more than a hundred and thirty visitors. The chairman for the first day was Prof. N. F. Mott (Cambridge), and for the second Prof. H. Welker (Siemens-Schuckertwerke, Erlangen, Germany). A total of nineteen papers was presented, mostly of a fundamental nature, with the emphasis on compounds of the zinc-blende type. In particular, the compound InSb, for which both the technology and theory are furthest advanced, received much attention. Arrangements have been made to publish the full papers in a special issue of the Journal of Electronics.

Three of the papers dealt with generalizations of a theoretical nature. Dr. F. Herman (R.C.A., Princeton), in a paper read by Dr. H. W. Leverenz, put forward a method for correlating the electronic band structure of zinc-blende type compounds with position in the periodic table of the elements concerned. In, for example, the isoelectronic sequence $\mathrm{Ge}_{\theta}$ (which may be regarded as the zinc-blende compound $\mathrm{GeGe}$ ), GaAs, ZnSe, CuBr, the crystal potential is regarded as primarily that of germanium with a small perturbing potential due to the ionic contribution in the binding, the strength $(s)$ of which increases in equal steps as we proceed along the sequence. For the regions of interest in momentum-space, the energy shifts are found to be quadratic in s. Measured values of the forbidden energy gap should show this same dependence, provided that no switch over occurs in the positions of the band edges in momentum-space. This latter possibility prevents outright prediction in the absence of supporting evidence, but the theory should be very useful in forming an early picture of the band structure in particular compounds of this type.

Similarly, C. H. L. Goodman (General Electric Co., Ltd., Wembley) offered a basis for the prediction of the electron mobility for a zinc-blende compound from a knowledge of the forbidden energy gap and the lattice spacing. He assumes that the covalent contribution to the binding energy and hence to the energy gap depends only on the interatomic distance (d) and is the same function of this as in the case of the Group IV elements. The excess of measured energy gap over that for a hypothetical Group IV element of the samo spacing is regarded as an ionic contribution $\left(E_{\text {ion }}\right)$. The quantity $\left(E_{\text {ion }} d^{3}\right)^{1 ! 2}$ should be closely related to the bond dipole, which in turn should control the mobility, both through its influence on the effective mass, as discussed by Seraphin, and through its effect on the scattering of electrons by optical modes of lattice vibration. A plot of existing data' on mobility against this parameter does give, in fact, a smooth curve, with a peak in the region of InSb, from which values for other compounds can be estimated with a fair amount of plausibility. 